\title{
Association analysis of COMT polymorphisms with schizophrenia and smooth pursuit eye movement abnormality
}

\author{
Byung Lae Park ${ }^{1}$, Hyoung Doo Shin ${ }^{1,2}$, Hyun Sub Cheong ${ }^{1}$, Chul Soo Park ${ }^{3}$, Jin-Wook Sohn ${ }^{3}$, Bong-Jo Kim ${ }^{3}$, \\ Han-Kil Seo ${ }^{3}$, Jae Won Kim ${ }^{4}$, Ki-Hoon Kim ${ }^{5}$, Tae-Min Shin ${ }^{5}$, Ihn-Geun Choi ${ }^{6}$, Shin Gyeom Kim ${ }^{7}$ \\ and Sung-Il $\mathrm{Woo}^{7}$
}

Schizophrenia is a multifactorial disorder characterized by the contribution of multiple susceptibility genes that may act in conjunction with epigenetic processes and environmental factors. The catechol- 0 -methyltransferase (COMT) gene, which is located in the 22q11 microdeletion, has been considered as a candidate gene for schizophrenia because of its ability to degrade catecholamines, including dopamine. In a genetic analysis, neurophysiological endophenotype in schizophrenia, such as smooth pursuit eye movement (SPEM) disturbance, is considered to be a good trait marker, because it may be under more direct genetic control. This study was performed to examine the genetic association of COMT polymorphisms with the risk of schizophrenia and SPEM abnormality in a Korean population. Six single-nucleotide polymorphisms of COMT were genotyped by TaqMan assay. Their genetic effects on the risk of schizophrenia were analyzed in 354 patients and 396 controls using $\chi^{2}$ analyses. Among the schizophrenic patients, 166 subjects were selected for association analyses of COMT polymorphisms with SPEM abnormality. From the six COMT polymorphisms, $r$ s6267 showed an association with the reduced risk of schizophrenia after correction $\left(P_{\text {corr }}=0.02\right)$. In analysis of SPEM abnormality, no significant associations were detected with COMT polymorphisms. The results of the present study provide the evidence that in a Korean population, COMT on the 22q11 locus is likely involved in the development of schizophrenia, but not in the SPEM function abnormality. Journal of Human Genetics (2009) 54, 709-712; doi:10.1038/jhg.2009.102; published online 30 October 2009

Keywords: COMT; schizophrenia; single-nucleotide polymorphism; smooth pursuit eye movement

\section{INTRODUCTION}

Schizophrenia (MIM 181500) is a multifactorial disorder characterized by the contribution of multiple susceptibility genes that may act in conjunction with epigenetic processes and environmental factors. ${ }^{1}$

The catechol-O-methyltransferase (COMT; MIM 116790) gene, which is located in the 22q11 microdeletion, has been considered as a candidate gene for schizophrenia because of its function of degrading catecholamines including dopamine. ${ }^{2}$ The Val158Met functional polymorphism among the COMT polymorphisms leads to a fourfold reduction in enzyme activity, ${ }^{3,4}$ and this variation of enzyme activity presumably affects the risk of schizophrenia. The associations between cognition in 22q11 deletion syndrome and COMT polymorphisms have been scrutinized in several studies, but the results have not been consistent and are as yet controversial. ${ }^{5-9}$

Schizophrenia is also recognized as a multifactorial disorder encompassing several intermediate phenotypes, each of which is likely affected by one or more genes. Defining and applying these endophenotypes in genetic studies is important for identifying schizophrenia-related genes, because they are likely under more direct genetic control. ${ }^{10}$ Smooth pursuit eye movement (SPEM) disturbance, which can be used as a neurophysiological endophenotype, may be found in $\sim 40-80 \%$ of patients with schizophrenia and $<10 \%$ of healthy control subjects. ${ }^{11}$ However, the genetic origin of the SPEM abnormality has been poorly understood, and relatively little research has been performed to answer these questions. The association of COMT rs4680 (G>A; Val158Met) with the SPEM abnormality in schizophrenia has been reported. ${ }^{10}$ Patients with the A/A (Met/Met) genotype of COMT rs4680 showed poorer predictive pursuit eye movement than did patients with the G/G (Val/Val) genotype, but normal controls with the A/A (Met/Met) genotype showed better predictive pursuit eye movement than did patients with the $\mathrm{G} / \mathrm{G}$ (Val/Val) genotype. In contrast, only male schizophrenia patients with the A (Met) allele showed less severity of

${ }^{1}$ Department of Genetic Epidemiology, SNP Genetics, Seoul, Republic of Korea; ${ }^{2}$ Department of Life Science, Sogang University, Seoul, Republic of Korea; ${ }^{3}$ Department of Psychiatry, College of Medicine, Gyeongsang National University, Jinju, GyeongsangNam Do, Republic of Korea; ${ }^{4}$ Division of Life Science and Research Institute of Life Science, Gyeongsang National University, Jinju, GyeongsangNam Do, Republic of Korea; ${ }^{5}$ Department of Biomedical Engineering, Yonsei University, Wonju, Republic of Korea; ${ }^{6}$ Department of Neuropsychiatry, Hallym University, Han-Gang Sacred Heart Hospital, Seoul, Republic of Korea and ${ }^{7}$ Department of Neuropsychiatry, Soonchunhyang University Hospital, Seoul, Republic of Korea

Correspondence: Dr S-I Woo, Department of Neuropsychiatry, Soonchunhyang University Hospital, 657, Hannam-dong, Yongsan-gu, Seoul, 140-743, Republic of Korea. E-mail: siwoo@hosp.sch.ac.kr

Received 31 May 2009; revised 27 September 2009; accepted 28 September 2009; published online 30 October 2009 
SPEM functional abnormality in another study, ${ }^{12}$ and subsequently no further study was performed to clarify this inconsistence.

In the present study, we analyzed the association of COMT polymorphisms with the risk of schizophrenia. We also analyzed the association of the SPEM abnormality with COMT polymorphisms among schizophrenic patients.

\section{MATERIALS AND METHODS}

\section{Subjects}

All individuals included in this study were of Korean ethnic origin; they consisted of 354 schizophrenia patients (176 males and 178 females) receiving stable doses of typical antipsychotic medications and 396 control subjects (224 males and 172 females). Schizophrenia patients were recruited from three Korean mental hospitals: the Keyo Hospital (Kyunggi-Do), the Jinju Mental Hospital (Jinju) and the Soonyoung Hospital (Gyeongsangnam Do). The age of the schizophrenic group was in the range of $23-76$ years (mean age $=44.0$ years; s.d. $=9.3$ ). The schizophrenia diagnoses were based on Diagnostic and Statistical Manual of Mental Disorders, 4th Edition (DSM-IV) criteria (American Psychiatric Association, 1994) ${ }^{13}$ These criteria were applied by two trained psychiatrists after an earlier clinical diagnosis of schizophrenia. Patients with complicating diagnoses of mental retardation, organic brain damage, drug or alcohol abuse, neurological disorders or autoimmune disorders, and those with low comprehension skills were excluded. Patients with drug side effects, such as tardive dyskinesia, extrapyramidal symptoms or oculogyric crisis, were also excluded from the study.

The population controls were unrelated healthy employees of the Center for Health Promotion of Seoul National University Hospital (Seoul, Korea), with an age range of $28-80$ years (mean age $=54.6$ years; s.d. $=9.3$ ). Each population control subject was evaluated by a trained clinician using the Structured Clinical Interview for DSM-IV, non-patient edition (SCID-NP), to ensure that the individual did not have an ongoing or previous psychiatric illness. The institutional review board of each hospital approved the study, and all subjects provided an informed consent.

\section{The measurement of SPEM}

We evaluated SPEM of schizophrenia patients by electrooculographic (EOG) recordings of eye movement and calculated the natural logarithmic values of the signal-to-noise ratio ( $\mathrm{Ln} \mathrm{S} / \mathrm{N}$ ratio). By this method, the global quantitative SPEM abnormality was scored and analyzed as the $\mathrm{Ln} \mathrm{S} / \mathrm{N}$ ratio, and the genetic association analysis was performed. A higher score on the $\mathrm{Ln} \mathrm{S} / \mathrm{N}$ ratio denotes good SPEM function and the lower score means poorer function. Subjects were seated in a darkened quiet room, in front of a 19-inch computer monitor at a distance of $40 \mathrm{~cm}$. Patients were instructed to observe the moving target spot, as closely as possible, during the pursuit task. The green target spot $(0.8 \mathrm{~cm} \times 1 \mathrm{~cm})$ appeared at the center of the computer monitor screen for $0.5 \mathrm{~s}$ and then moved horizontally back and forth in an 18.2 degree of visual arc to each side of the screen at a constant speed of 28.2 degree per sec. Using Biopac MP150 (BIOPAC Systems, Goleta, CA, USA), the electrophysiological analog signals of SPEM were amplified and sampled at $400 \mathrm{~Hz}$ and converted into digitized files. A measurement period of $15 \mathrm{~s}$ during the SPEM task was resampled at $4 \mathrm{~Hz}$ and passed through a $2 \mathrm{~Hz}$ low-pass filter. After application of Fast Fourier Transformation to the EOG data, the $\mathrm{Ln} \mathrm{S} / \mathrm{N}$ ratio was calculated from analysis of the power spectrum curves.

A total of 166 schizophrenia patients ( 81 males and 85 females) were analyzed for SPEM. Among them, each set of 87, 52 and 27 patients were recruited from the Keyo Hospital, the Jinju Mental Hospital and the Soonyoung Hospital, respectively. Subjects were selected among patients who agreed to participate and were able to understand the procedure of eye-tracking task. Schizophrenia patients were then divided into two groups for statistical analysis based on their SPEM function. The Ln S/N ratio (mean \pm s.d.) of the good and poor SPEM function groups was $4.35 \pm 0.29$ and $3.20 \pm 0.70$, respectively.

\section{Single-nucleotide polymorphism genotyping}

Among the COMT polymorphisms previously examined in other studies, ${ }^{14-16}$ six single-nucleotide polymorphisms (SNPs) were selected for validation in the Korean population, using the criteria of the International HapMap database, from over $10 \%$ of minor allele frequencies (MAFs) in the Asian population.
The selected SNPs were genotyped using the TaqMan ${ }^{17}$ assay. Genotyping quality control was performed in $10 \%$ of the samples by duplicate checking (rate of concordance in duplicates $>99 \%$ ). The sequences and detailed descriptions of the primers used are summarized in Supplementary Table 2.

\section{Statistics}

We examined Lewontin's $D^{\prime}\left(\left|D^{\prime}\right|\right)$ and the linkage disequilibrium (LD) coefficient $r^{2}$ between all pairs of bi-allelic loci. ${ }^{18}$ Haplotypes were inferred using the algorithm, Haploview, developed by the Broad Institute (Cambridge, MA, USA). ${ }^{19} \chi^{2}$-Tests were used to compare the observed numbers of each genotype with those expected for the population under Hardy-Weinberg equilibrium (HWE). The allelic distributions of polymorphisms and haplotypes among patients with schizophrenia and normal subjects were evaluated by $\chi^{2}$-analyses (two-tailed). Multiple regressions were also used for association analyses of SPEM reliability. To correct for multiple testing, the effective number of independent markers was calculated using the software SNPSpD (http://genepi.qimr.edu.au/general/daleN/SNPSpD/), which is based on the spectral decomposition $(\mathrm{SpD})$ of matrices of pair-wise $\mathrm{LD}$ values between SNPs. ${ }^{20}$ The resulting number of independent marker loci of COMT (4.9998) was applied to correct for multiple testing. Statistical power of single associations was calculated with false-positive rate of $5 \%$, disease lifetime prevalence of $0.4 \%,{ }^{21}$ given MAFs and sample sizes and assuming a relative risk of 1.3 , using PGA (Power for Genetic Association Analyses) software. ${ }^{22}$

\section{RESULTS}

In this study, we analyzed the association of COMT polymorphisms with the risk of schizophrenia and SPEM abnormality using $\chi^{2}$-tests. Six polymorphisms were selected among previously studied SNPs reported in the previous literatures. ${ }^{14-16}$ The distribution of rs6267 in patients and rs4680 in controls significantly deviated from HWE (Supplementary Table 1). LDs among SNPs were measured by calculating Lewontin's $D^{\prime}$ and $r^{2}$ values (Supplementary Figure 1). With the exception of rs165599 because of its low linkage with other SNPs, analysis showed that five polymorphisms were parsed into two blocks with each block having storing LD spine. There were three major haplotypes (MAF $>5 \%)$ in each block. COMT BL1_ht1, BL1_ht2, BL2_ht2 and BL2_ht3 were not analyzed, because they were almost identical to rs740603, rs737865, rs4633 and rs6267, respectively. Haplotypes with MAF less than $5 \%$ were also excluded in further analysis.

In the initial analysis for the risk of schizophrenia, COMT rs6267 showed an association with the reduced risk of schizophrenia $\left(P=0.004\right.$ and $P_{\text {corr }}=0.02$, Table 1$)$ (MAF of patients versus controls; 0.068 versus 0.110 , respectively). In the analysis of abnormality of SPEM quality, although rs165599 showed marginal association with schizophrenia and abnormality of SPEM quality, no significant associations were detected (Table 2).

\section{DISCUSSION}

Velo-cardio-facial syndrome is known to be caused by the microdeletion of the 22q11 locus, ${ }^{23}$ and many studies have focused on the association between genes on the microdeletion locus of 22q11 and schizophrenia. COMT has been an attractive candidate gene for schizophrenia because of its biological function, including degradation of catecholamines, and the gene's location in the locus. ${ }^{2}$ The COMT Val-158 allele of rs4680 has garnered particular attention regarding susceptibility to schizophrenia, because its physiological effect on prefrontal information processing might add to or interact with other causes of prefrontal malfunction for those at risk for schizophrenia. ${ }^{24}$ Although COMT has been established as a candidate gene for the risk of schizophrenia, the association of COMT polymorphisms is still controversial. In an Ashkenazi Jewish population, rs737865 and rs165599 rather than rs4680 (Val158Met), showed significant 
Table 1 Association analysis of COMT polymorphisms with the risk of schizophrenia in a Korean population

\begin{tabular}{|c|c|c|c|c|c|c|c|c|c|}
\hline \multirow[b]{2}{*}{ Loci } & \multirow[b]{2}{*}{ Position } & \multirow[b]{2}{*}{ Amino acid change } & \multirow[b]{2}{*}{ Allele } & \multicolumn{2}{|c|}{ Allele distribution } & \multirow[b]{2}{*}{$\chi^{2}$} & \multirow[b]{2}{*}{$\mathrm{P}$ of $\chi^{2}$} & \multirow[b]{2}{*}{$P_{\text {corr }}$} & \multirow[b]{2}{*}{ Statistical power (\%) } \\
\hline & & & & SzO & $P C$ & & & & \\
\hline rs737865 T>C & & & C & 217 (30.7\%) & 232 (29.4\%) & & & & \\
\hline \multirow[t]{2}{*}{ rs740603 A>G } & Intron1 & & A & $377(54.2 \%)$ & 435 (55.3\%) & 0.206 & 0.65 & 1 & 94.75 \\
\hline & & & G & $319(45.8 \%)$ & 351 (44.7\%) & & & & \\
\hline \multirow[t]{2}{*}{$\mathrm{rs} 6267 \mathrm{G}>\mathrm{T}$} & Exon3 & A72S & G & $660(93.2 \%)$ & 701 (89.0\%) & 8.242 & 0.004 & 0.02 & 65.84 \\
\hline & & & $\mathrm{T}$ & $48(6.8 \%)$ & 87 (11.0\%) & & & & \\
\hline \multirow[t]{2}{*}{$\mathrm{rs} 4680 \mathrm{G}>\mathrm{A}$} & Exon4 & M158V & G & 495 (70.7\%) & $583(74.2 \%)$ & 2.222 & 0.14 & 0.68 & 89.94 \\
\hline & & & A & 205 (29.3\%) & 203 (25.8\%) & & & & \\
\hline \multirow[t]{2}{*}{ rs165599 A>G } & $3^{\prime}$ & & A & $398(57.2 \%)$ & $410(52.2 \%)$ & 3.751 & 0.05 & 0.26 & 94.76 \\
\hline & & & G & $298(42.8 \%)$ & 376 (47.8\%) & & & & \\
\hline
\end{tabular}

Abbreviations: COMT, catechol-O-methyltransferase; MAF, minor-allele frequency; PC, population control; $P_{\text {corr, }}$ corrected $P$-value; SNP, single-nucleotide polymorphism; SZO, schizophrenia patient. Haplotypes were constructed using five SNPs, including rs737865T $>C$, rs740603A $>$ G, rs4633C $>$ T, rs6267G $>$ T and rs4680G $>A$. rs $165599 A>G$ was not used in haplotype construction because of its low linkage with other SNPs. BL1_ht1, BL1_ht2, BL2_ht2 and BL2_ht3 were not analyzed because they were almost identical to rs740603, rs737865, rs4633 and rs6267, respectively. Haplotypes with below $5 \%$ of MAF were also excluded in further analysis. To achieve the optimal correction for multiple testing of SNPs in linkage disequilibrium (LD) with each other, the effective number of independent marker loci (4.9998) in COMT was calculated using the software SNPSpD (http://genepi.qimr.edu.au/general/daleN/SNPSpD/), on the basis of the spectral decomposition (SpD) of matrices of pair-wise LD between SNPs.

Table 2 Association analysis of COMT polymorphisms with SPEM abnormality in two SPEM categories among Korean schizophrenic patients

\begin{tabular}{|c|c|c|c|c|c|c|c|}
\hline Loci & Allele & Poor SPEM & Good SPEM & $\chi^{2}$ & $\mathrm{P}$ of $\chi^{2}$ & $P_{\text {corr }}$ & Statistical power (\%) \\
\hline rs737865 T>C & C & $53(31.2 \%)$ & 55 (34.0\%) & & & & \\
\hline \multirow[t]{2}{*}{ rs740603 A>G } & $A$ & $84(49.4 \%)$ & 79 (48.8\%) & 0.014 & 0.91 & 1 & 39.24 \\
\hline & G & $86(50.6 \%)$ & $83(51.2 \%)$ & & & & \\
\hline \multirow[t]{2}{*}{ rs6267 G>T } & G & $154(90.6 \%)$ & $155(95.7 \%)$ & 3.177 & 0.07 & 0.37 & 11.24 \\
\hline & $\mathrm{T}$ & $16(9.4 \%)$ & $7(4.3 \%)$ & & & & \\
\hline \multirow[t]{2}{*}{$\mathrm{rs} 4680 \mathrm{G}>\mathrm{A}$} & G & $126(74.1 \%)$ & $116(71.6 \%)$ & 0.264 & 0.61 & 1 & 34.59 \\
\hline & $A$ & 44 (25.9\%) & $46(28.4 \%)$ & & & & \\
\hline \multirow[t]{2}{*}{ rs165599 A>G } & $A$ & $83(48.8 \%)$ & $99(61.1 \%)$ & 5.042 & 0.02 & 0.12 & 38.46 \\
\hline & G & 87 (51.2\%) & $63(38.9 \%)$ & & & & \\
\hline
\end{tabular}

Abbreviations: COMT, catechol-O-methyltransferase; Ln S/N ratio, logarithmic values of the signal-to-noise ratio; $P_{\text {corr }}$, corrected $P$-value; SPEM, smooth pursuit eye movement. Good and Poor SPEM groups were divided at the Ln S/N ratio of 3.97.

association with the risk of schizophrenia. ${ }^{15}$ On the other hand, no COMT polymorphism was found to affect schizophrenia in the European, Japanese and Chinese populations. ${ }^{16,25}$ In previous studies conducted in the Korean population, COMT rs4680 was reported to have no genetic effect on schizophrenia, ${ }^{14,26}$ whereas rs6267 seemed to be a genetic factor for the risk of schizophrenia. ${ }^{14}$ Lee et al. reported that the frequency of the Tallele of rs6267 (Ala72Ser) was significantly higher in the Korean schizophrenia patients than in control subjects (10.2 versus $8.2 \%)$. On the other hand, a study conducted in the Japanese population reported that the significant association between this SNP and schizophrenia could not be found, for example, $10.2 \%$ in patients versus $8.2 \%$ in control subjects, ${ }^{27}$ and $8.7 \%$ in patients versus $8.6 \%$ in control subjects. ${ }^{28}$ It is hard to expound on the discrepancies among the results of the studies at this stage. However, if the relative risk was small and/or MAF was low, the possibility of a type II error as a result of insufficient statistical power might be possible. Further studies should be performed using larger samples in several populations.

Except for rs6267 in cases and rs4680 in controls (Supplementary Table 1), all the SNPs in this study were consistent with HWE. Although it would be hard to decipher the cause of deviation of rs4680 $(P=0.040)$, deviation of rs6267 could be attributed to association with the disease. In population-based case-control studies, there 
is a possibility that the disease group may deviate from HWE because of selection bias. If there is indeed a gene-disease association, cases are not necessarily expected in HWE. Therefore, the deviation from HWE could be an additional evidence for the true association of this marker.

SPEM is initiated by the motion of an object's image across the retina, and smooth pursuit is generated to minimize the velocity error between the moving image and the fovea. ${ }^{29}$ It has been proposed that two kinds of motion information processing, retinal perceptive motion signal processing and extraretinal predictive motion signal processing, drive SPEM. The extraretinal motion-processing deficit is thought to be directly related to the eye-tracking abnormality in schizophrenia. ${ }^{30,31}$ Our method of measuring the SPEM function is a global measurement of the SPEM, which is adequate for genetic analysis. We were thus able to quantitatively calculate the degree of SPEM impairment based on the $\mathrm{Ln}$ S/N ratio of the SPEM curves. Schizophrenia patients were then divided into two groups, 'good' and 'poor,' according to their SPEM function using the $\mathrm{Ln} \mathrm{S} / \mathrm{N}$ ratio value of 3.97. As there were $\sim 40-80 \%$ schizophrenic patients reported to have an incidence of SPEM, dividing the schizophrenia patients into two groups according to their SPEM function was justified under the hypothesis that $50 \%$ of patients have SPEM disturbance. In addition, we did not perform the association analysis of the SPEM function abnormality with COMT polymorphisms in normal controls. When considering the low incidence of SPEM impairment in normal controls $(<10 \%)$, the association analysis in normal control subjects could not retain statistical power.

In the recent genetic association studies of the COMT rs 4680 (G $>$ A) with SPEM abnormality, ${ }^{10}$ schizophrenia patients with the A/A genotype (Met/Met) showed poorer predictive pursuit performance than those with $\mathrm{G} / \mathrm{G}$ genotype (Val/Val). Interestingly, it has been suggested that in a gender-specific manner, COMT polymorphisms might be associated with eye-tracking abnormality in schizophrenia. ${ }^{12}$ However, there was no significant association detected between COMT polymorphisms and the abnormality of SPEM quality in our subjects (Supplementary Table 3). Our result in this study regarding this inconsistency did not favor any previous report, because we found no association of COMT polymorphisms with SPEM severity in schizophrenia patients.

In summary, we scrutinized the association of six SNPs and four major haplotypes of COMT with the risk of schizophrenia and the abnormality of SPEM quality among schizophrenic patients in a Korean population. There was a significant association between COMT rs6267 and the reduced risk of schizophrenia after correction $\left(P_{\text {corr }}=0.02\right)$; however, no association was detected between COMT polymorphisms and SPEM abnormality in schizophrenia.

\section{CONFLICT OF INTEREST}

The authors declare no conflict of interest.

1 Kirov, G., O’Donovan, M. C. \& Owen, M. J. Finding schizophrenia genes. J. Clin. Invest. $115,1440-1448$ (2005).

2 Axelrod, J. \& Tomchick, R. Enzymatic O-methylation of epinephrine and other catechols. J. Biol. Chem. 233, 702-705 (1958).

3 Chen, J., Lipska, B. K., Halim, N., Ma, Q. D., Matsumoto, M., Melhem, S. et al. Functional analysis of genetic variation in catechol-O-methyltransferase (COMT): effects on mRNA, protein, and enzyme activity in postmortem human brain. Am. J. Hum. Genet. 75, 807-821 (2004).

4 Lotta, T., Vidgren, J., Tilgmann, C., Ulmanen, I., Melen, K., Julkunen, I. et al. Kinetics of human soluble and membrane-bound catechol O-methyltransferase: a revised mechanism and description of the thermolabile variant of the enzyme. Biochemistry 34, 4202-4210 (1995).

5 Shashi, V., Keshavan, M. S., Howard, T. D., Berry, M. N., Basehore, M. J., Lewandowski, E. et al. Cognitive correlates of a functional COMT polymorphism in children with $22 \mathrm{q} 11.2$ deletion syndrome. Clin. Genet. 69, 234-238 (2006).

6 Kates, W. R., Antshel, K. M., Abdulsabur, N., Colgan, D., Funke, B., Fremont, W. et al. A gender-moderated effect of a functional COMT polymorphism on prefrontal brain morphology and function in velo-cardio-facial syndrome (22q11.2 deletion syndrome). Am. J. Med. Genet. B Neuropsychiatr. Genet. 141B, 274-280 (2006).

7 Bearden, C. E., Jawad, A. F., Lynch, D. R., Sokol, S., Kanes, S. J., McDonaldMcGinn, D. M. et al. Effects of a functional COMT polymorphism on prefrontal cognitive function in patients with 22q11.2 deletion syndrome. Am. J. Psychiatry 161, 1700-1702 (2004).

8 Baker, K., Baldeweg, T., Sivagnanasundaram, S., Scambler, P. \& Skuse, D. COMT Val108/158 Met modifies mismatch negativity and cognitive function in 22q11 deletion syndrome. Biol. Psychiatry 58, 23-31 (2005).

9 Glaser, B., Debbane, M., Hinard, C., Morris, M. A., Dahoun, S. P., Antonarakis, S. E. et al. No evidence for an effect of COMT Val158Met genotype on executive function in patients with 22q11 deletion syndrome. Am. J. Psychiatry 163, 537-539 (2006).

10 Thaker, G. K., Wonodi, I., Avila, M. T., Hong, L. E. \& Stine, O. C. Catechol O-methyltransferase polymorphism and eye tracking in schizophrenia: a preliminary report. Am. J. Psychiatry 161, 2320-2322 (2004).

11 Holzman, P. S. Eye movements and the search for the essence of schizophrenia. Brain Res. Brain Res. Rev. 31, 350-356 (2000).

12 Rybakowski, J. K., Borkowska, A., Czerski, P. M. \& Hauser, J. Eye movement disturbances in schizophrenia and a polymorphism of catechol-0-methyltransferase gene. Psychiatry Res. 113, 49-57 (2002).

13 American Psychiatric Association. Diagnostic and Statistical Manual of Mental Disorders. 4th edn. (Wasington DC, American Psychiatric Association, 1994).

14 Lee, S. G., Joo, Y., Kim, B., Chung, S., Kim, H. L., Lee, I. et al. Association of Ala72Ser polymorphism with COMT enzyme activity and the risk of schizophrenia in Koreans. Hum. Genet. 116, 319-328 (2005).

15 Shifman, S., Bronstein, M., Sternfeld, M., Pisante-Shalom, A., Lev-Lehman, E., Weizman, A. et al. A highly significant association between a COMT haplotype and schizophrenia. Am. J. Hum. Genet. 71, 1296-1302 (2002).

16 Williams, H. J., Glaser, B., Williams, N. M., Norton, N., Zammit, S., MacGregor, S. et al. No association between schizophrenia and polymorphisms in COMT in two large samples. Am. J. Psychiatry 162, 1736-1738 (2005).

17 Livak, K. J. Allelic discrimination using fluorogenic probes and the $5^{\prime}$ nuclease assay. Genet. Anal. 14, 143-149 (1999).

18 Hedrick, P. W. Gametic disequilibrium measures: proceed with caution. Genetics 117, 331-341 (1987).

19 Barrett, J. C., Fry, B., Maller, J. \& Daly, M. J. Haploview: analysis and visualization of LD and haplotype maps. Bioinformatics 21, 263-265 (2005).

20 Nyholt, D. R. A simple correction for multiple testing for single-nucleotide polymorphisms in linkage disequilibrium with each other. Am. J. Hum. Genet. 74, 765-769 (2004).

21 Saha, S., Chant, D., Welham, J. \& McGrath, J. A systematic review of the prevalence of schizophrenia. PLoS Med. 2, e141 (2005).

22 Menashe, I., Rosenberg, P. S. \& Chen, B. E. PGA: power calculator for case-control genetic association analyses. BMC Genet. 9, 36 (2008).

23 Murphy, K. C. Schizophrenia and velo-cardio-facial syndrome. Lancet 359, 426-430 (2002).

24 Egan, M. F., Goldberg, T. E., Kolachana, B. S., Callicott, J. H., Mazzanti, C. M., Straub, R. E. et al. Effect of COMT Val108/158 Met genotype on frontal lobe function and risk for schizophrenia. Proc. Natl Acad. Sci. USA 98, 6917-6922 (2001).

25 Fan, J. B., Chen, W. Y., Tang, J. X., Li, S., Gu, N. F., Feng, G. Y. et al. Family-based association studies of COMT gene polymorphisms and schizophrenia in the Chinese population. Mol. Psychiatry 7, 446-447 (2002).

26 Kim, Y. R., Kim, J. H., Kim, S. J., Lee, D. \& Min, S. K. Catechol-O-methyltransferase Val158Met polymorphism in relation to aggressive schizophrenia in a Korean population. Eur. Neuropsychopharmacol. 18, 820-825 (2008).

27 Nunokawa, A., Watanabe, Y., Muratake, T., Kaneko, N., Koizumi, M. \& Someya, T. No associations exist between five functional polymorphisms in the catecholO-methyltransferase gene and schizophrenia in a Japanese population. Neurosci. Res. 58, 291-296 (2007).

28 Okochi, T., Ikeda, M., Kishi, T., Kawashima, K., Kinoshita, Y., Kitajima, T. et al. Meta-analysis of association between genetic variants in COMT and schizophrenia: an update. Schizophr. Res. 110, 140-148 (2009).

29 Lisberger, S. G. \& Westbrook, L. E. Properties of visual inputs that initiate horizontal smooth pursuit eye movements in monkeys. J. Neurosci. 5, 1662-1673 (1985).

30 Hong, L. E., Mitchell, B. D., Avila, M. T., Adami, H., McMahon, R. P. \& Thaker, G. K. Familial aggregation of eye-tracking endophenotypes in families of schizophrenic patients. Arch. Gen. Psychiatry 63, 259-264 (2006).

31 Hong, L. E., Tagamets, M., Avila, M., Wonodi, I., Holcomb, H. \& Thaker, G. K. Specific motion processing pathway deficit during eye tracking in schizophrenia: a performance-matched functional magnetic resonance imaging study. Biol. Psychiatry 57, 726-732 (2005).

Supplementary Information accompanies the paper on Journal of Human Genetics website (http://www.nature.com/jhg) 